\title{
Spectrum of early lung cancer presentation in low-dose screening CT: a pictorial review
}

\author{
Cristiano Rampinelli ${ }^{1} \cdot$ Sonia Francesca Calloni ${ }^{2}$ • \\ Marta Minotti $^{2} \cdot$ Massimo Bellomi $^{1,2}$
}

Received: 27 October 2015 / Revised: 20 January 2016 / Accepted: 18 March 2016 /Published online: 17 May 2016

(C) The Author(s) 2016. This article is published with open access at Springerlink.com

\begin{abstract}
The typical presentation of early stage lung cancers on low-dose CT screening are non-calcified pulmonary nodules. However, there is a wide spectrum of unusual focal abnormalities that can be early presentations of lung cancer. These abnormalities include, for example, cancers associated with 'cystic airspaces' or scar-like cancers. The detection of lung cancer with low-dose CT can be affected by the absence of intravenous contrast medium. As a consequence, endobronchial and central lesions can be difficult to recognize, raising the potential for missed cancers. Focal lesions arising within pre-existing lung disease, such as lung fibrosis or apical scars, can also be early lung cancer manifestations and deserve particular consideration as recognition of these lesions may be hindered by the underlying disease. Furthermore, the unpredictable growth rate of lung cancer, which ranges from indolent to aggressive cancers, necessitates attention to the wide spectrum of progression in lung cancer appearance on serial lowdose CT scans. In this pictorial review we discuss the spectrum of early lung cancer presentation in low-dose CT screening, highlighting typical as well as unusual radiological features and the varied growth rates of early lung cancer.
\end{abstract}

Cristiano Rampinelli

cristiano.rampinelli@ieo.it

1 Department of Medical Imaging and Radiation Sciences, European Institute of Oncology, Via Ripamonti, 435, 20141 Milan, Italy

2 School of Medicine, University of Milan, Milan, Italy
Teaching Points

- There is a wide spectrum of early presentations of lung cancer on LDCT.

- Low radiation dose and the absence of contrast medium injection can affect lung cancer detection.

- Lung cancer growth shows various behaviours, ranging from indolent to aggressive cancers.

- Familiarity with LDCT technique can improve CT screening effectiveness and avoid missed diagnosis.

Keywords Lung cancer $\cdot$ Low-dose CT $\cdot$ Screening · Pulmonary nodule

\section{Introduction}

Lung cancer is the most commonly diagnosed cancer, and was the leading cause of cancer death globally in males in 2008; among females, it was the fourth most commonly diagnosed cancer and the second leading cause of cancer death [1].

The National Lung Cancer Screening Trial (NLST) has recently demonstrated that low-dose computed tomography (LDCT) reduces lung cancer mortality by $20 \%$ in high-risk subjects compared to chest X-ray screening [2]. Based on this result, LDCT screening of heavy smokers has recently been recommended by the major American and European scientific societies [3-6].

LDCT of the lung would seem to be a simple radiological interpretation but the absence of contrast material injection and low signal-to-noise ratio due to the low radiation exposure can lead to misinterpretation and missed significant findings.

Moreover, the distinction between different types of pulmonary nodules, awareness of the behaviours of early stage lung cancer and the interpretation of serial LDCT scans are fundamental in the management of the findings. 
In this pictorial review, we discuss the wide spectrum of early LDCT findings in lung cancer screening, highlighting typical as well as unusual radiological features and the varied growth rates of early stage lung cancer.

\section{Low-dose CT protocol}

LDCT for screening purposes is an unenhanced, single breathhold scan, conducted from the lung apices through the lung bases.

Low-dose protocols of the chest can be achievable due to a high contrast resolution between the air and lung nodules, gaining a low radiation dose while maintaining good diagnostic quality.

There is no consensus on which level of dose is considered a 'low-dose' CT, however, the major screening trials used tube voltages between $90 \mathrm{kVp}$ and $140 \mathrm{kVp}$ and tube current in the range of 30-80 mAs [7]. Both parameters should be combined to obtain an acceptable radiation dose, taking into account the patient's body habitus and age. The American College of Radiology (ACR), suggest a CTDIvol $\leq 3 \mathrm{mGy}$ for standard size patients [8] while an NLST study concluded that acceptable CT screening can be accomplished at an overall average effective dose of approximately $2 \mathrm{mSv}$ [9]. The National Comprehensive Cancer Care Network guidelines have suggested an effective dose of $\leq 3 \mathrm{mSv}$ for patients with a body mass index (BMI) $\leq 30 \mathrm{~kg} / \mathrm{m} 2$ and $\leq 5 \mathrm{mSv}$ for patients with a BMI $>30 \mathrm{~kg} / \mathrm{m} 2[10]$.

A gantry rotation time of $<0.5$ seconds is recommended by the ACR [8]: faster rotation is associated with fewer motion artifacts and a decreased radiation dose to the patient.

LDCT collimation should be set with the purpose of achieving thin-section reconstruction images, of at least $\leq 1.5 \mathrm{~mm}$ [8]. Thin-section images are essential in reading and interpretation of low-dose CT scans, allowing the use of a CAD system, volumetric analysis and a more accurate evaluation of subsolid nodules.

Most LDCT screening studies have used the filtered back projection technique for image reconstruction; nevertheless, iterative reconstruction algorithms have been shown to improve image quality while reducing radiation dose [11-13]. Recent studies have shown that iterative reconstruction algorithms allow the use of low-dose CT and ultra-low-dose CT with acceptable image quality $[14,15]$ and that the detection of subsolid nodules is not affected by such algorithms $[14,16]$.

Various methods and tools are available in reporting chest low-dose CT. Beside the evaluation of axial thin-section images, the detection rate of pulmonary nodules can be improved using maximum intensity projection (MIP) which consists of projecting the voxel with the highest attenuation value on every view throughout the volume onto a $2 \mathrm{D}$ image. Bastarrika et al. [17] showed that non-overlapping, 10-mmthick axial MIP reconstructions enabled more accurate detection of pulmonary nodules in comparison with 1.25 $\mathrm{mm}$ conventional axial images. Furthermore, Jankowski et al. evaluated the diagnostic benefits of a MIP and CAD system for pulmonary nodule detection compared with 1mm LDCT images and found that MIP and CAD reduced the number of overlooked nodules [18]. Also, multiplanar reconstruction images (MPR) were shown to be a sensitive technique in lung nodule detection [19], which can be particularly useful for evaluation of lung hilum and apex.

The use of CAD systems can improve radiologists detection rate for small pulmonary nodules [20]. The accuracy of $\mathrm{CAD}$ for nodule detection increases with thinner reconstruction images and with overlapping reconstructed sections [21], even if this causes an increasing number of false-positive results.

However, to date, there is no consensus on the optimal reading technique; further investigations are needed to assess which method should be used to improve the detection rate of pulmonary nodules on low-dose CT.

\section{Lung cancer presenting as lung nodules}

Low-dose CT can identify small pulmonary nodules in heavy smokers and thus improve the detection of lung cancer at an earlier and potentially more curable stage $[22,23]$. Noncalcified pulmonary nodules are the typical presentation of screen-detected lung cancers on low-dose CT. Based on the definition of the Fleischner Society [24], the pulmonary nodule is a rounded or irregular opacity, well or poorly defined, measuring up to $3 \mathrm{~cm}$ in diameter.

According to their attenuation, lung nodules can be classified as solid or subsolid, the latter further divided into partsolid or non-solid nodules [25].

The spectrum of early stage lung cancer presentation at low-dose CT includes solid nodules as well as part-solid nodules and ground-glass nodules (Fig. 1).

A pulmonary nodule is defined as solid when it completely obscures the entire lung parenchyma within it. Non-solid nodules, or pure ground-glass nodules (pGGN), are defined as focal nodular areas of increased attenuation through which lung parenchymal structures, such as the pulmonary vessels or airway structures, can be visualized. Part-solid nodules are those having both ground-glass and solid components.

Lung nodule attenuation is, therefore, a key feature in lung cancer screening, with particular emphasis on subsolid nodules. Subsolid nodules are complex lesions both for interpretation and management, and recently the Fleischner society has published recommendations for their management [26]. Of subsolid nodules, 40-70\% resolve spontaneously in 3 months and, upon resolution, are presumed to have been benign [27]. Part-solid nodules that persist at 3-month follow-up should be considered potentially malignant: in the ELCAP study, after adjusting for nodule size, the malignancy rates 


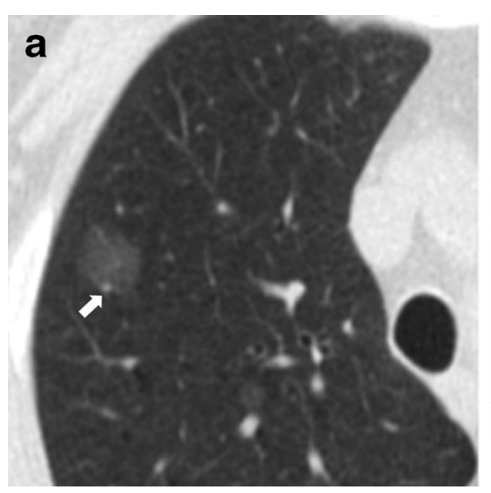

Fig. 1 Primary lung adenocarcinomas. Radiological features of pulmonary nodules according to their attenuation. (a) A low-dose CT thin-section shows a $15-\mathrm{mm}$ non-solid nodule (pure ground-glass attenuation) of the right upper lobe: the nodule density is slightly higher than that of normal lung parenchyma, and vessels are visualized within the nodule (arrows). (b) Low-dose CT scan shows a 25-mm part-solid nodule of the right lower lobe. This nodule is characterized by a $3-\mathrm{mm}$
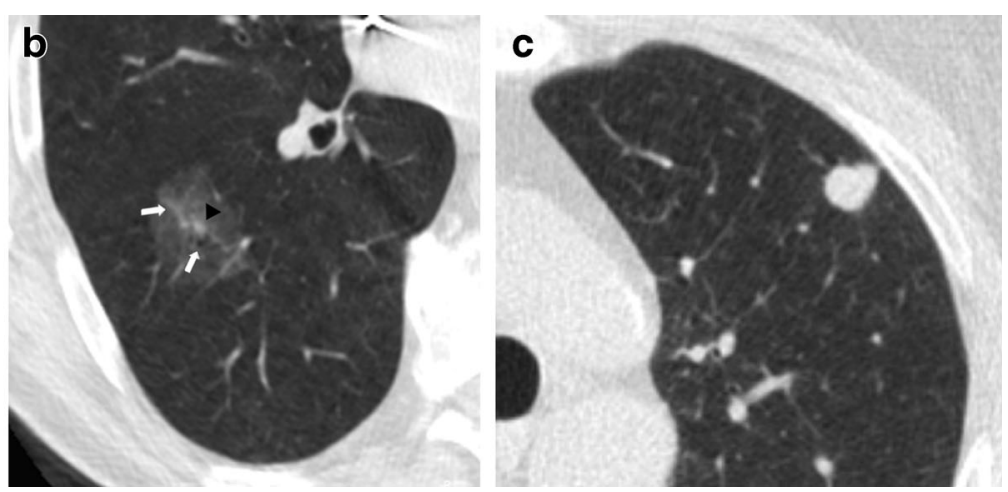

central solid component (arrowhead) surrounded by a pure ground-glass opacity. The vessels and a small peripheral airway (arrow) can be clearly depicted within the ground-glass opacity. (c) Axial low-dose CT scan shows a 12-mm subpleural solid nodule of the left upper lobe. The spectrum of early stage lung cancer presentation at low-dose CT includes solid nodules as well as part-solid nodules and ground-glass nodules

(NELSON) trial. The authors found that participants with a nodule smaller than $5 \mathrm{~mm}$ had a very low risk $(0.6 \%)$ of developing lung cancer during 2 years of follow-up.

An increase in pulmonary nodule attenuation at LCDT follow-up, the development or increment of a solid component within the pulmonary nodule should raise suspicions of malignancy, even if the nodule size is stable (Figs. 2 and 3).

The pulmonary nodule size is another important predictor for malignancy in heavy smokers, as good evidence exists for a strong correlation between nodule size and risk of malignancy $[28,29]$ : in a meta-analysis of eight CT screening trials, the prevalence of malignancy depended on nodule size, ranging from $0 \%$ to $1 \%$ for nodules $<5 \mathrm{~mm}$, from $6 \%$ to $28 \%$ for those between 5 and $10 \mathrm{~mm}$, and from $64 \%$ to $82 \%$ for nodules $>20 \mathrm{~mm}$ [30]. A recent study by Horeweg et al. [31] reported the probability of developing lung cancer in participants from the Dutch-Belgian Lung Cancer Screening

\section{Unusual presentations of lung cancer}

Besides the typical presentation of lung cancer, e.g. pulmonary nodule, there is a wide spectrum of focal abnormalities seen on low-dose CT that could not truly be defined as a pulmonary nodules but, nevertheless, are early presentations of primary lung cancer. These kinds of abnormalities, which are unusual forms compared to pulmonary nodules, include cancers associated with 'cystic airspaces' or 'non-nodular' cancers presenting, for example, as fibrotic changes or scarlike lesions. Such focal abnormalities are initial signs of lung cancer which usually develop over time in a lung nodule or
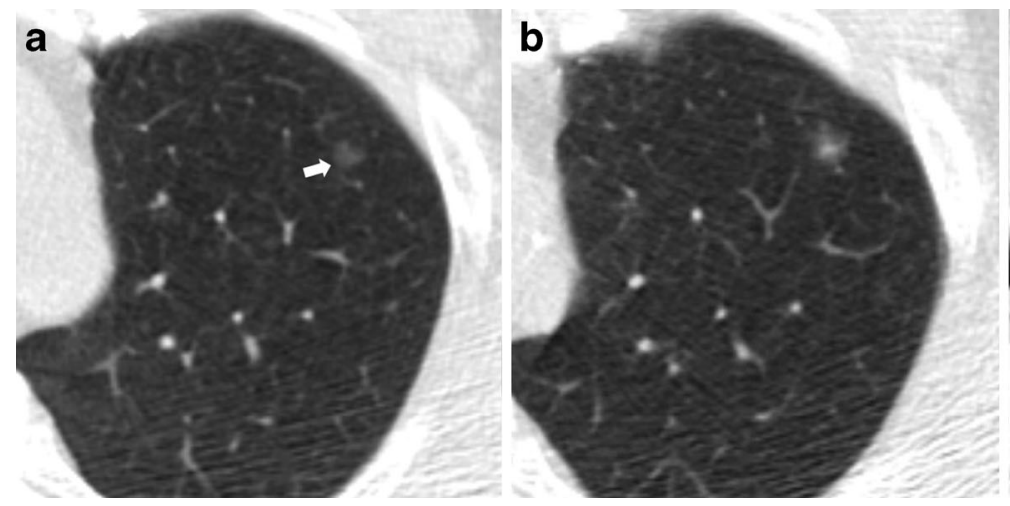

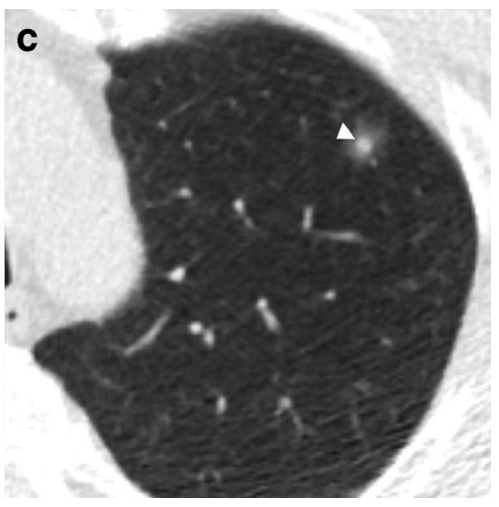

Fig. 2 Increased attenuation of a small lung adenocarcinoma over 3 years in a 62-year-old man. (a) Low-dose CT image show a 5-mm nonsolid nodule of the upper left lobe (arrow). (b) Follow-up CT obtained 1 year later show a minimum increment of axial diameter and a slight increase of nodule attenuation. (c) Two years later the progression to a part-solid nodule is obvious. The development of a central solid component (arrowhead) within the nodule can be observed. An increase in pulmonary nodule attenuation at LCDT follow-up, the development or increment of a solid component within the pulmonary nodule should raise suspicions of malignancy, even if the nodule size is stable. Typically, this appearance is suggestive of a peripheral lung adenocarcinoma 


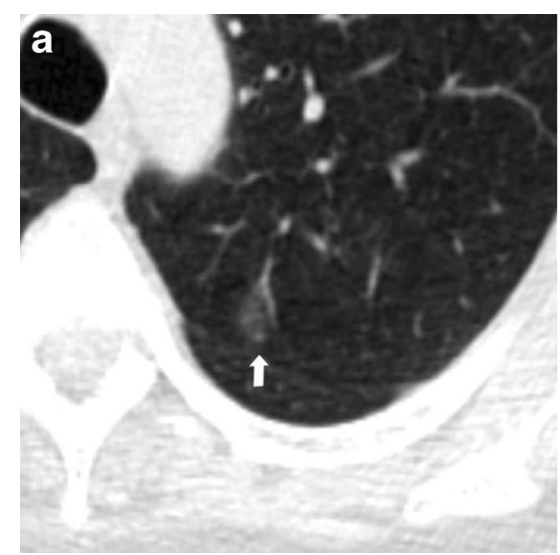

Fig. 3 Progression at the 1-year interval of a non-solid nodule in a 66year-old woman. (a) Low-dose CT section shows a 12-mm nodule with ground-glass attenuation of the upper left lobe (arrow). (b) CT scan obtained at annual repeat LDCT demonstrates a substantial stability in

suspicious pulmonary opacities and, therefore, should be checked at follow-up or annual repeat LDCT.

There is increasing evidence that early stage lung cancer could arise from pre-existing pulmonary 'cystic airspaces' [32-35], including in this description all findings associated with air spaces, such as cysts, bullae, and blebs.

These cancers associated with cystic airspaces have recently been described in a lung cancer screening program [36]: out of 26 lung cancers identified abutting or in the wall of a cystic airspace, 13 were identified at baseline $(13 / 595,2 \%)$ and 13 at annual screening $(13 / 111,12 \%)$, which was significant $(p<$ 0.0001 ). In a recent analysis aimed at evaluating findings of 22 interval and post-screen carcinomas in lung cancer screening, the authors showed that $22 \%(5 / 22)$ of missed cancers presented as bulla wall thickening on LDCT [37, 38].

This kind of presentation is usually slow growing on serial low-dose CT scans [26] and the diagnosis of lung cancer is suggested by the circumferential wall thickening of the cystic

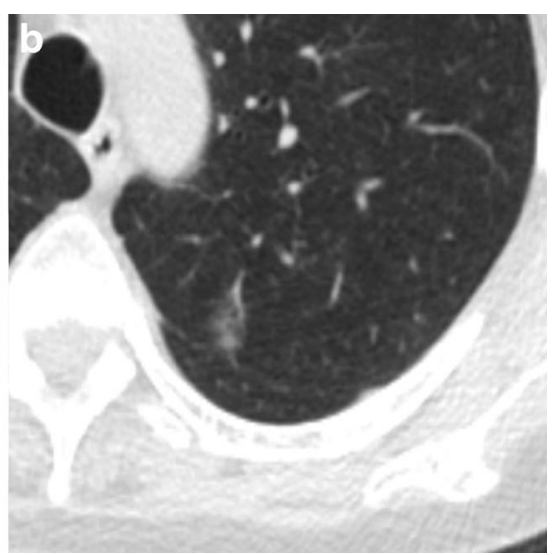

size but with increased density, that indicates an increased risk for malignancy. The lesion was surgically removed with a diagnosis of lung adenocarcinoma. Sub-solid nodules that show an increment in attenuation should be considered suspicious for malignancy

airspaces or by the arising of a nodule along the wall of the cystic airspaces (Fig. 4). Nevertheless, with tumor growth, airspaces size could increase, decrease or remain unchanged [35].

Lung cancer presenting as fibrotic changes or as scarlike lesions are less common but could be encountered in LDCT screening (Fig. 5). In a recent article aimed at evaluating the early features of incident lung cancers detected with LDCT [39], a remarkable percentage (7/17, 41 $\%)$ of cancers which exhibited an initial 'non-nodular' shape were observed. Similarly, Xu et al. [40] reviewed the images of patients with diagnoses of lung cancer in annual repeat rounds of $\mathrm{CT}$ screening and showed that some cancers were recognized at previous LDCT scans but they were considered to represent benign rather than malignant findings. In that study, the cancers were misclassified as fibrosis, scarring, and a simple bulla, which the authors attributed to a lack of experience in
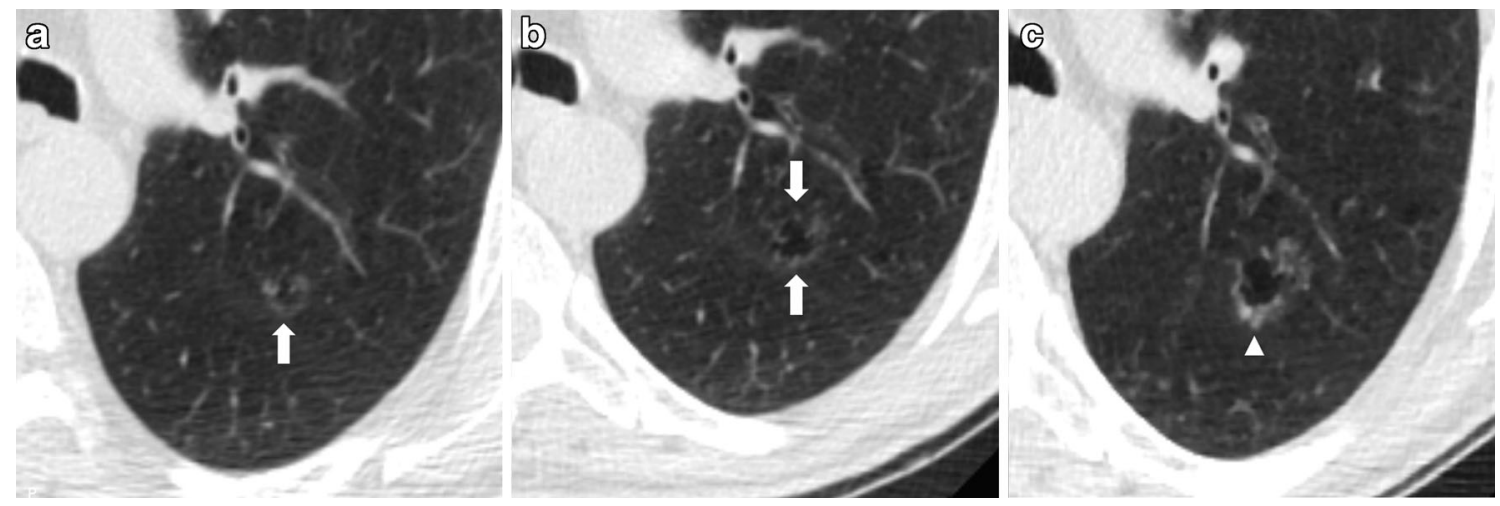

Fig. 4 Progressive changes of a lung adenocarcinoma associated with a cystic airspace in a 60-year-old woman. (a) Axial low-dose CT shows an 8-mm cystic airspace with thin irregular non-solid wall in the upper left lobe (arrow). (b) One year later the lesion has increased in size to $12 \mathrm{~mm}$ and 2 years later, (c) a consistent irregular thickening with the development of solid components along the wall was evident (arrowhead). The histologic diagnosis was lung adenocarcinoma. This kind of presentation is usually slow growing on serial low-dose CT scans and the diagnosis of lung cancer is suggested by the circumferential wall thickening of the cystic airspaces; with tumor growth, airspaces size could increase, decrease or remain unchanged 
Fig. 5 Low-dose CT images show both an increase in size and density of a scar-like lesion of the left lung apex, consisting of a lung adenocarcinoma. (a, c) Axial and coronal LDCT sections of the left apex show an irregular subpleural opacity that was interpreted as apical fibrotic changes. $(\mathbf{b}, \mathbf{d})$ LDCT repeated at a 1-year interval shows spiculations (arrowhead) and a significant thickening of the lesion (arrow). A PET/CT scan was positive and the patient went to lobectomy with histologic diagnosis of adenocarcinoma. Lung cancers presenting as fibrotic changes or as scar-like lesions are relatively uncommon but could be encountered in LDCT screening

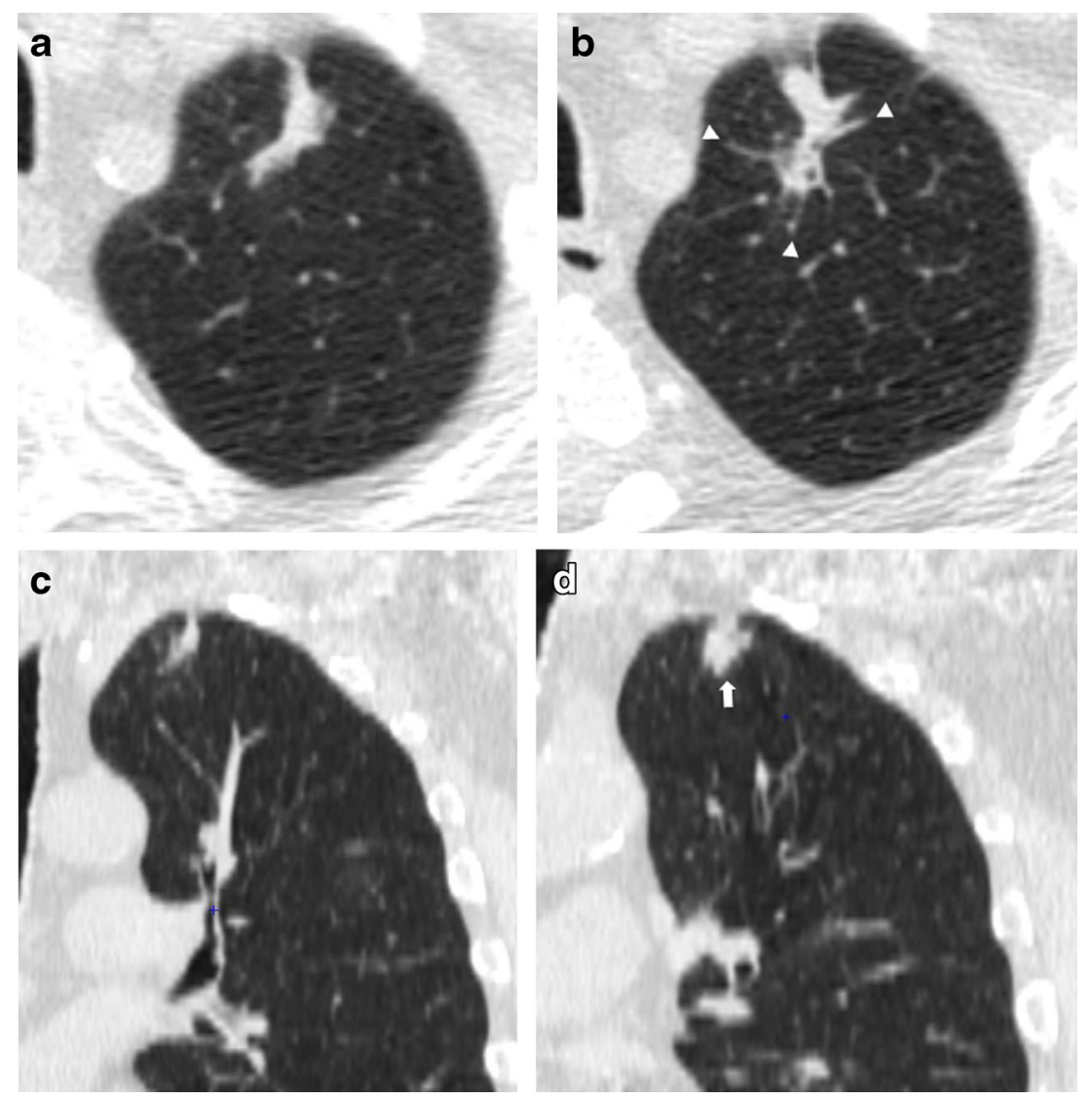

identifying findings suspicious for small lung cancers or identifying small cancer with an unusual appearance.

\section{Lung cancer growth}

One of the advantages in lung cancer screening is the availability of serial annual LDCT scans, permitting comparison of early changes of suspicious findings.

The growth rate of CT screen-detected lung cancer is often unpredictable, with many characteristics such as size, morphology, and attenuation that should be taken into account managing suspicious lung nodules. Early lung cancers could present various behaviours at LDCT follow-up, ranging from very slow growth to rapidly growing cancers.

Growth rate can be difficult to estimate and volumetric analyses of pulmonary nodules has been suggested as a precise tool for nodule size measurement and an accurate method for assessing nodule growth. In the NELSON trial, solid intraparenchymal nodules were measured by volumetric software and with semi-automated measurements, the interobserver agreement was excellent: identical volumes were measured by both observers in $89 \%$ of nodules. However, in a minority (approximately $11 \%$ ) of small solid nodules, semiautomated measurements were not completely reproducible and, thus, may cause errors in the assessment of nodule growth. The most frequent cause of variability was incomplete segmentation performed by the software due to an irregular shape or irregular margins of the nodule. For this reason, for small or irregularly shaped nodules, an observer should check the segmentation shown by the program before accepting the semiautomated volume measurement and drawing conclusions about growth or stability of a nodule [41].

Obviously, the behaviours of screen-detected lung cancer is strongly related to its histology: small cells and squamous cell lung cancers are usually more aggressive forms compared to adenocarcinomas which have a wide range of growth but are usually slower [42].

Tipically, early lung cancer presenting as non-solid nodules (ground-glass nodules, GGN) are slow-growing forms. Such cancers, even if nodule size is determinant, could have such indolent behaviours that short-term LDCT follow-up (e.g. 3 months) could be insufficient to determinate the real growth, and longer follow-ups (e.g. up to 3 years) are desirable to assess nodule growth (Fig. 6) [26]. When growth is very slow and previous LDCT scans are available, it is necessary to make annual comparisons with the oldest LDCT scan available to carefully note slight changes over time.

Part-solid nodules, especially those in which the solid component is larger than $5 \mathrm{~mm}$, should be considered malignant until proven otherwise, provided either growth or no change is 

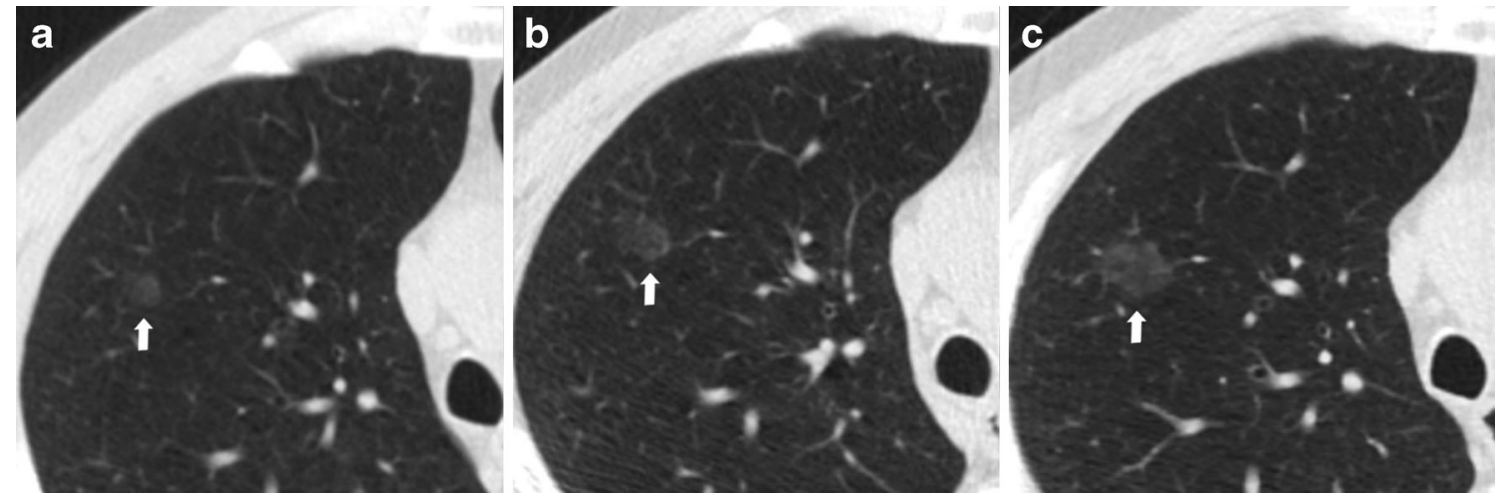

Fig. 6 Slow-growing lung adenocarcinoma in a 61-year-old man. (a) Low-dose CT axial section in the right upper lobe shows a 6-mm ground-glass nodule (arrow). (b) Low-dose CT scan after 2 years shows an increase in size of the nodule to $10 \mathrm{~mm}$ (arrow). (c) After 4

seen at the 3-month LDCT follow-up . For these complex lesions, the growth of the solid portion is more important and reliable in assessing the malignancy of the nodule (Fig. 2).

Solid nodules have a wide range of growth but usually present faster growth compared to subsolid nodules [43, 44] (Fig. 7).

It is also important to consider that care should be taken not to assume that all lesions that partially decrease in size at short-term LDCT follow-up are necessarily benign. It is documented that adenocarcinomas can decrease temporarily in size after antibiotic therapy, owing to fibrosis or atelectasis [26]; in this case, a further LDCT scan is suggested to assess the complete resolution or stability of the findings and to avoid missed diagnosis (Fig. 8).

\section{LDCT: lesion location and cancer detection}

The detection of lung cancer with low-dose CT may be hindered by the absence of intravenous contrast medium and the low signal-to-noise ratio. As a consequence, endobronchial lesions, pulmonary-hilum lung cancers, and parenchymal abnormalities at lung apices or within lung fibroses are difficult to recognize, raising the potential for missed cancers. years from the first low-dose CT scan, the nodule showed the same ground-glass attenuation but a progressive increase in size from 10 to $14 \mathrm{~mm}$ (arrow). Typically, early lung cancer presenting as non-solid nodules are slow-growing forms

Even if small lung cancer detected by screening are more frequently localised in peripheral lung areas, nevertheless, a small amount of lung cancers are also centrally located or

The majority of screen-detected lung cancers are localised in the periphery of the lungs, represented by the large amount of adenocarcinomas more often localised peripherally [31], while squamous cell and small cell carcinomas are most common centrally. A retrospective study performed by Lindell and colleagues [45] showed that squamous cell and small cell carcinomas represented $71 \%$ and $88 \%$, respectively, of central lung lesions ( $11 \%$ of cancers detected).

Compared to peripherally located carcinomas, such lung cancers could be difficult to detect because low-dose CT images are unenhanced scans and possibly because screening a population at this location is far less common. In a recent publication by Scholten and colleagues [46] focused on missed lung cancer in the NELSON screening trial, the authors showed that more than one-third of the 22 missed cancers were endobronchial lesions $(5 / 22)$ or mediastinal/ hilar lymph nodes (3/22). A dedicated evaluation of endobronchial and hilar regions, with the use of coronal MPR and both lung and mediastinal window settings, is, endobronchial.
Fig. 7 Fast-growing lung adenocarcinoma in a 79-year-old man. (a) Axial LDCT image of the chest shows a 7-mm subpleural solid nodule in the left upper lobe (arrow). (b) LDCT scan obtained at 1-year interval shows a rapid growth of the lesion into a 23-mm solid nodule (arrow) with an aggressive behavior documented by the concomitant enlargement of hylar lymph nodes (arrowheads)
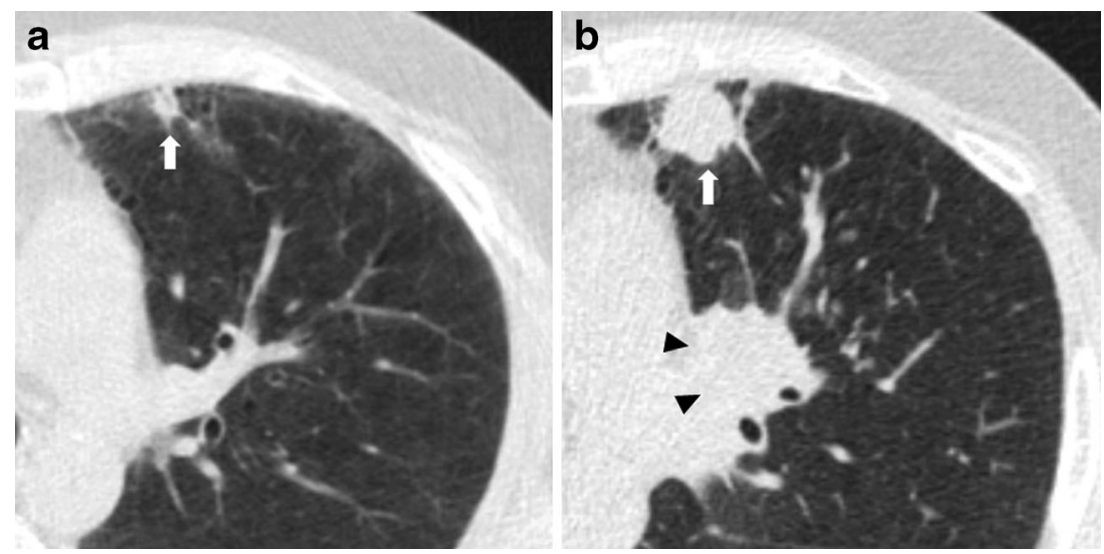

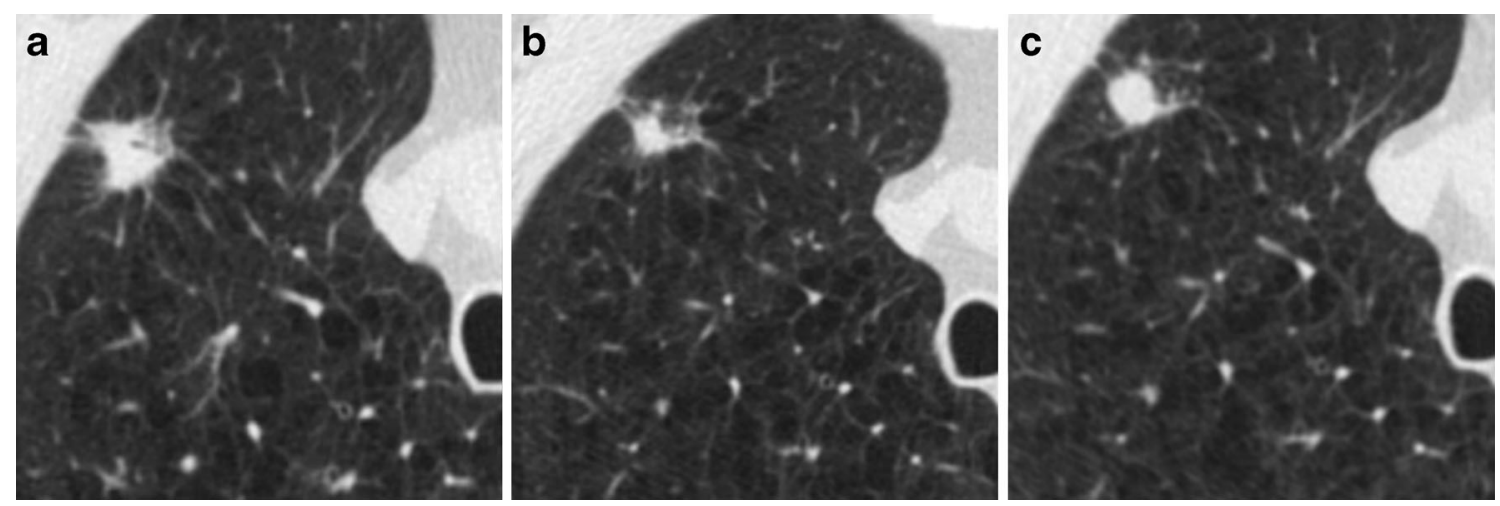

Fig. 8 Transient decrease in size of a squamous cell lung cancer in a 74year-old man. (a) Unenhanced axial LDCT image of the chest shows a solid nodule in the upper right lobe with an irregular shape and a maximum diameter of $19 \mathrm{~mm}$. (b) Follow-up LDCT scan obtained 1 month after antibiotic therapy demonstrated a significant regression of the lesion. (c) At LDCT obtained 3 months later, the nodule grew in size

therefore, suggested to minimize detection errors in lung cancer screening (Figs. 9 and 10). Furthermore, endobronchial and central lesions are mostly represented by fast-growing or fast-progressing tumors such as squamous cell carcinomas and small cell carcinomas [47] with a usual VDT shorter than adenocarcinoma's [48], making the early diagnose of these lesions a worthy challenge, as these rapidly growing missed cancers have the potential to affect patient prognoses. again. Biopsy was performed, and results of histology revealed lung adenocarcinoma. It is reported that adenocarcinomas can show a transient decrease in size, maybe related to fibrosis or atelectasis. In this cases, a further LDCT scan is suggested to assess the complete resolution or stability of the findings and to avoid missed diagnosis

Additional lung regions that deserve specific consideration in lung cancer screening are the lung apices. The detection and interpretation of parenchymal abnormalities within lung apices could be a challenge in lung cancer screening, as apical regions are a frequent location of fibrotic scars and differential diagnosis between lung cancer and fibrotic changes could be complicated. Indeed, as reported by Scholten and colleagues [46], an apical scar-like nodule was identified as a cause of a missed lung cancer due to an interpretation error. Similarly,
Fig. 9 Lung cancer of the left hylum in a 67-year-old man with history of sarcoidosis. (a) Axial low-dose CT image through lung hyla does not show any significant findings at baseline CT screening. (b) Low-dose CT image obtained at the same level 1 year later shows the appearance of a 6-mm pulmonary nodule (arrow) surrounded by vascular and airways structures of the left hylum. This finding without contrast material injection can be misinterpreted as a pulmonary vessel. (c) The following year, the low-dose CT scan reveals the presence of a large lesion of the left hylum (arrows) that was characterize by increased metabolic activity via PET scan (d). The histological diagnosis was a T2N1 squamous cell carcinoma
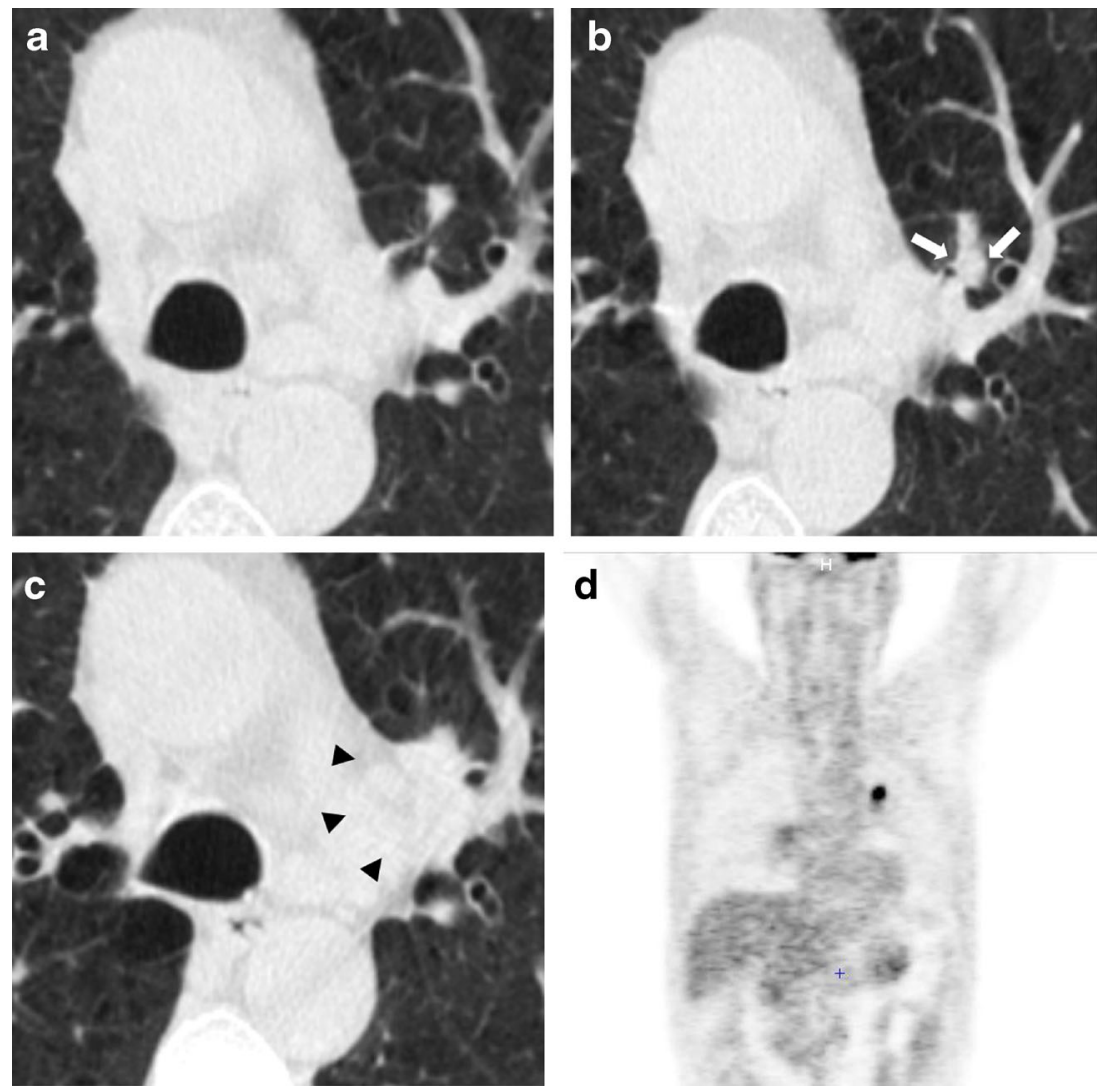
Fig. 10 Endobronchial lung cancer. (a) Axial low-dose CT shows a small lesion abutting the posterior wall of the right main bronchus, interpreted as nonsuspicious bronchial mucus (arrow). (b) Low-dose CT performed 1 year later shows a significant reduction of bronchial caliber due to the presence of neoplastic tissue (arrowheads)
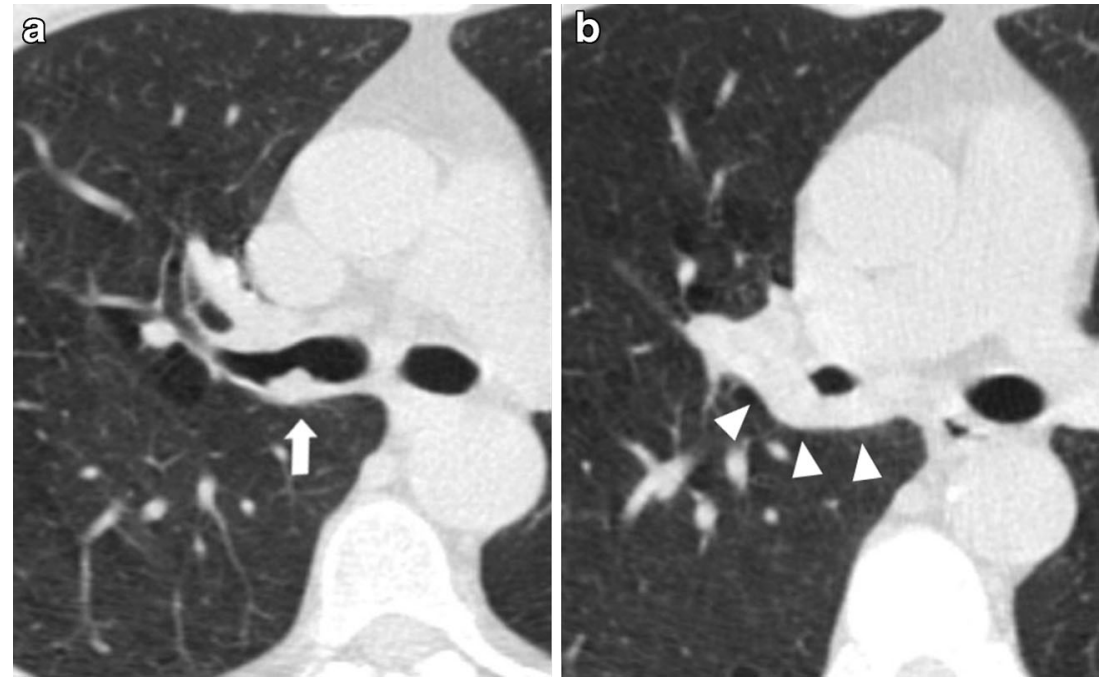

Diederich and colleagues [49] reported one nodule interpreted as an apical scar at initial LDCT that showed slow growth after 24 months was revealed to be lung adenocarcinoma.

Lung cancer diagnosis should, therefore, be taken into account when a new lesion arises in a previously detected apical scar or when a focal apical abnormality shows progressive enlargement at annual repeat low-dose CT scans. Any change over time within an apical scarring should be carefully evaluated to detect potential lung cancer (Fig. 11).

The detection of lung cancer could also be affected by the location of the lesion when arising within previous lung abnormalities, such as interstitial lung disease. Sverzellati and colleagues [50] showed that important abnormalities consistent with fibrotic interstitial lung disease may be encountered in participants in a lung cancer screening trial, with the crude prevalence of usual interstitial pneumonia/other chronic interstitial pneumonia (UIP/OCIP) of $4.0 \%$ (95 \% CI 2.8-5.9\%). In the NELSON Screening trial, signs of pulmonary fibrosis were reported in $117(8 \%)$ out of 1409 cases [51]. The presence of radiological signs of interstitial lung disease could delay the diagnosis of early stage lung cancer as tumors can be hidden or overlooked in the presence of underlying lung abnormalities (Fig. 12). This is even more important if we consider that idiopathic pulmonary fibrosis is associated with an increased risk of
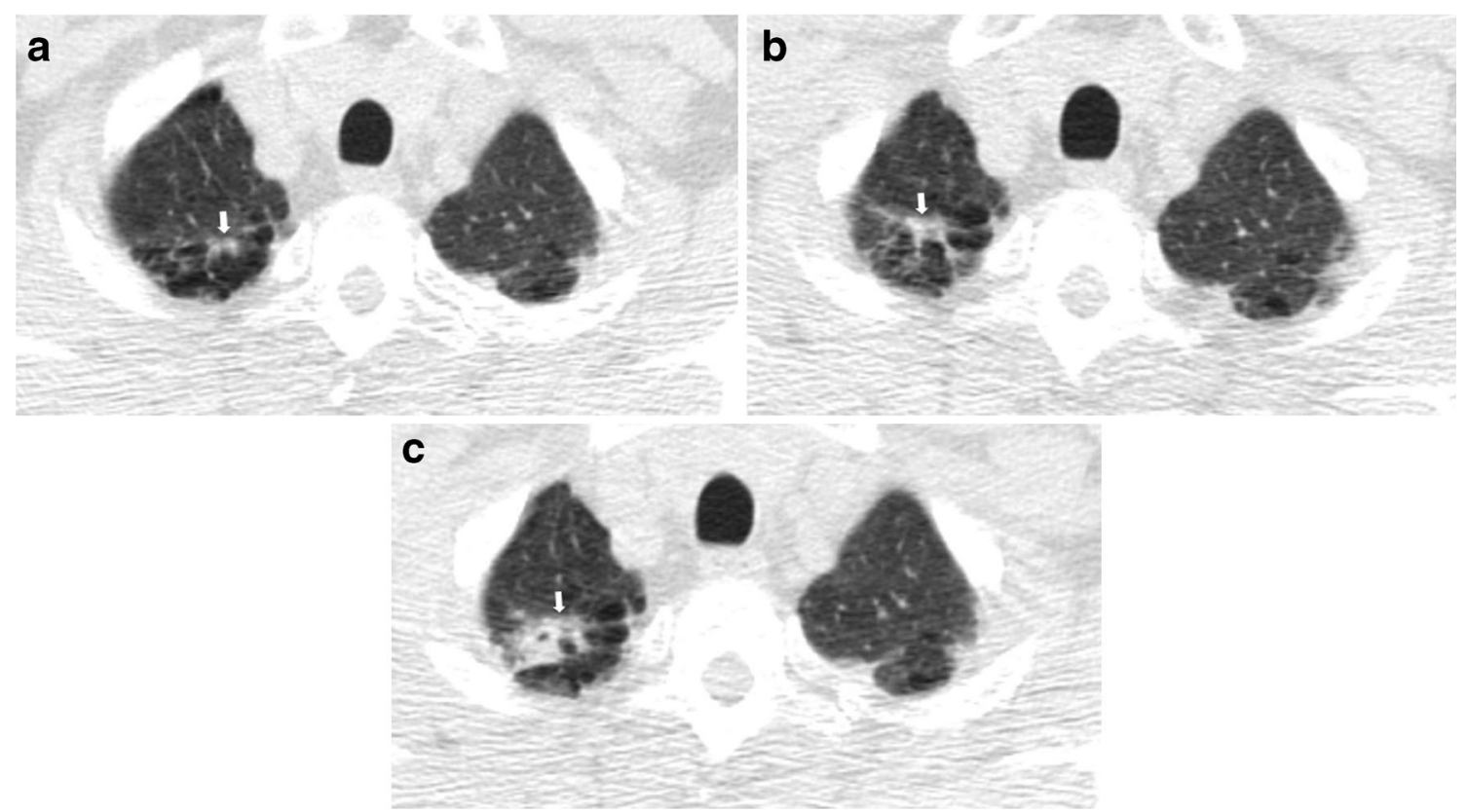

Fig. 11 Right apical lung cancer in a 71-year-old man. (a) Low-dose axial CT scan shows small irregular fibrotic changes of the right apex (arrow). (b) One year later, LDCT shows a slight increment of the focal abnormalities with the typical aspect of apical fibrotic changes, with a pleural tag and concomitant emphysema (arrow). (c) Two years later, a remarkable increment in size was clearly depicted (arrow) and the lesion has been surgically removed with a diagnosis of lung adenocarcinoma 

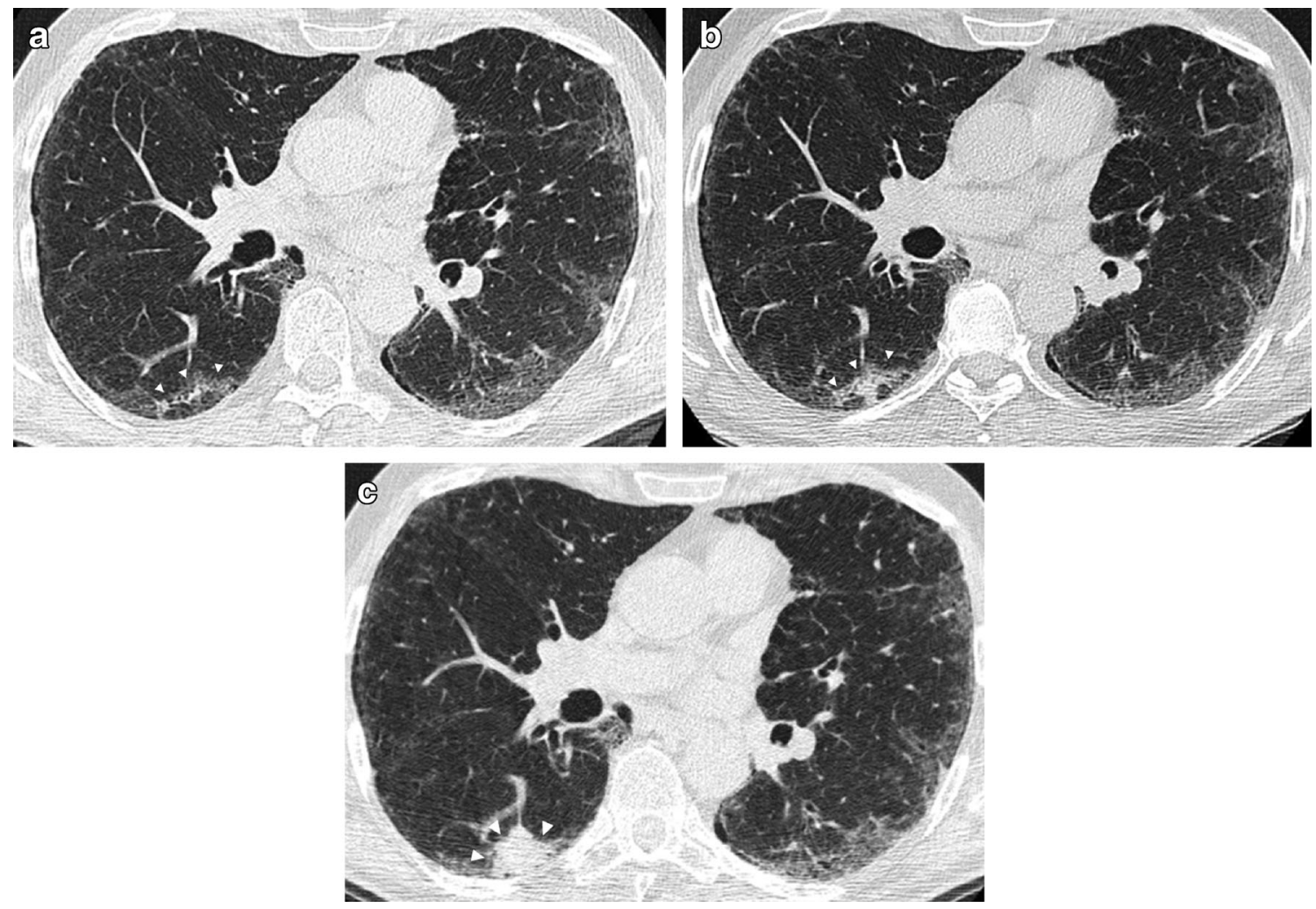

Fig. 12 Lung cancer arising within a pre-existing fibrotic lung disease. (a) Low-dose CT demonstrates a bilateral subpleural reticular pattern and a ground-glass opacity, with a subpleural curvilinear opacity in the right lower lobe (arrowheads). (b) Low-dose CT scan obtained 1 year later confirmed the fibrotic disease with an increased density of the subpleural

lung cancer [52] and some studies reported that the tumors preferentially occurred in areas with lung fibrosis [53, 54].

\section{Conclusion}

There is a wide spectrum of low-dose CT findings in lung cancer screenings. Awareness of the various radiological features of early lung cancers and the familiarity with the lowdose CT technique can improve the CT screening effectiveness and avoid missed diagnosis.

Open Access This article is distributed under the terms of the Creative Commons Attribution 4.0 International License (http:// creativecommons.org/licenses/by/4.0/), which permits unrestricted use, distribution, and reproduction in any medium, provided you give appropriate credit to the original author(s) and the source, provide a link to the Creative Commons license, and indicate if changes were made.

\section{References}

1. Jemal A, Bray F, Center MM et al (2011) Global cancer statistics. CA Cancer J Clin 61:69-90 opacity in the right lower lobe (arrowheads), interpreted as a progression of the fibrosing pattern. (c) At follow-up CT performed 1 year later, there is a remarkable increase of subpleural opacity in the right lobe with the development of a 32-mm mass, resulting in lung adenocarcinoma (arrow)

2. Aberle DR, Adams AM, Berg CD, Black WC, Clapp JD, Fagerstrom RM, Gareen IF, Gatsonis C, Marcus PM, Sicks JD, National Lung Screening Trial Research Team (2011) Reduced lung-cancer mortality with low-dose computed tomographic screening. N Engl J Med 365(5):395-409

3. Detterbeck FC, Mazzone PJ, Naidich DP, Bach PB (2013) Screening for lung cancer: Diagnosis and management of lung cancer, 3rd ed: American College of Chest Physicians evidencebased clinical practice guidelines. Chest 143(5 Suppl):78S-92S

4. Jaklitsch MT, Jacobson FL, Austin JH, Field JK, Jett JR, Keshavjee S, MacMahon H, Mulshine JL, Munden RF, Salgia R, Strauss GM, Swanson SJ, Travis WD, Sugarbaker DJ (2012) The American Association for Thoracic Surgery guidelines for lung cancer screening using low-dose computed tomography scans for lung cancer survivors and other high-risk groups. J Thorac Cardiovasc Surg 144(1):33-38

5. Wender R, Fontham ET, Barrera E Jr, Colditz GA, Church TR, Ettinger DS, Etzioni R, Flowers CR, Gazelle GS, Kelsey DK, LaMonte SJ, Michaelson JS, Oeffinger KC, Shih YC, Sullivan DC, Travis W, Walter L, Wolf AM, Brawley OW, Smith RA (2013) American Cancer Society lung cancer screening guidelines. CA Cancer J Clin 63(2):107-117

6. Kauczor HU, Bonomo L, Gaga M, Nackaerts K, Peled N, Prokop M, Remy-Jardin M, von Stackelberg O, Sculier JP, European Society of Radiology (ESR) and the European Respiratory Society (ERS) (2015) ESR/ERS white paper on lung cancer screening. Eur Respir J 46(1):28-39

7. Rampinelli C, Origgi D, Bellomi M (2013) Low-dose CT: technique, reading methods and image interpretation. Cancer Imaging 12:548-556 
8. http://www.acr.org/ /media/ACR/Documents/PDF/QualitySafety/ Lung \% 20 Screening/Lung \% 20 Cancer \% 20 Screening \% 20Technical\%20Specifications.pdf Accessed online 05-05-2015.

9. Larke FJ, Kruger RL, Cagnon CH et al (2011) Estimated radiation dose associated with low-dose chest CT of average-size participants in the National Lung Screening Trial. AJR 197:1165-1169

10. National Comprehensive Cancer network guidelines version (2015). Available at: http://www.nccn.org/professionals/ physician_gls/pdf/lung_screening.pdf

11. Hu XH, Ding XF, Wu RZ et al (2011) Radiation dose of nonenhanced chest CT can be reduced $40 \%$ by using iterative reconstruction in image space. Clin Radiol 66:1023-1029

12. Pontana F, Duhamel A, Pagniez J, Flohr T, Faivre JB, Hachulla AL et al (2011) Chest computed tomography using iterative reconstruction vs filtered back projection (Part 2): image quality of low-dose CT examinations in 80 patients. Eur Radiol 21:636-643

13. Leipsic J, Nguyen G, Brown J, Sin D, Mayo JR (2010) A prospective evaluation of dose reduction and image quality in chest CT using adaptive statistical iterative reconstruction. AJR Am J Roentgenol 195:1095-1099

14. Rampinelli C, Origgi D, Vecchi V, Funicelli L, Raimondi S, Deak P, Bellomi M (2015) Ultra-low-dose CT with model-based iterative reconstruction (MBIR): detection of ground-glass nodules in an anthropomorphic phantom study. Radiol Med 120:611-617

15. Kim Y, Kim YK, Lee BE, Lee SJ, Ryu YJ, Lee JH, Chang JH (2015) Ultra-Low-Dose CT of the Thorax Using Iterative Reconstruction: Evaluation of Image Quality and Radiation Dose Reduction. AJR Am J Roentgenol 204(6):1197-1202

16. Siegelman JW, Supanich MP, Gavrielides MA (2015) Pulmonary nodules with ground-glass opacity can be reliably measured with low-dose techniques regardless of iterative reconstruction: results of a phantom study. AJR Am J Roentgenol 204(6):1242-1247. doi:10. 2214/AJR.14.13820

17. Bastarrika Aleman G, Dominguez Echavarri PD, Noguera Tajadura JJ, Arraiza Sarasa M, Zudaire Diaz-Tejeiro B, Zulueta Frances J (2008) Usefulness of maximum intensity projections in lowradiation multislice CT lung cancer screening. Radiol 50:231-237

18. Jankowski A, Martinelli T, Timsit JF et al (2007) Pulmonary nodule detection on MDCT images: evaluation of diagnostic performance using thin axial images, maximum intensity projections, and computer-assisted detection. Eur Radiol 17:3148-3156

19. Eibel R, Turk TR, Kulinna C, Herrmann K, Reiser MF (2011) Multidetector-row CT of the lungs: Multiplanar reconstructions and maximum intensity projections for the detection of pulmonary nodules. Röfo 173:815-821

20. Rubin GD, Lyo JK, Paik DS et al (2005) Pulmonary nodules on multidetector row CT scans: performance comparison of radiologists and computer-aided detection. Radiol 234:274-283

21. Kim JS, Kim JH, Cho G, Bae KT (2005) Automated detection of pulmonary nodules on CT images: effect of section thickness and reconstruction interval-initial results. Radiol 236:295-299. doi: 10.1148/radiol.2361041288

22. Henschke CI, McCauley DI, Yankelevitz DF, Naidich DP, McGuinness G, Miettinen OS, Libby DM, Pasmantier MW, Koizumi J, Altorki NK, Smith JP (1999) Early Lung Cancer Action Project: overall design and findings from baseline screening. Lancet 354(9173):99-105

23. Veronesi G, Maisonneuve P, Spaggiari L, Rampinelli C, Pardolesi A, Bertolotti R, Filippi N, Bellomi M (2014) Diagnostic performance of low-dose computed tomography screening for lung cancer overfive years. J Thorac Oncol 9(7):935-939

24. Hansell DM, Bankier A, MacMahon $\mathrm{H}$ et al (2008) Fleischner society: glossary of terms for thoracic imaging. Radiol 246:697722

25. Henschke CI, Yankelevitz DF, Mirtcheva R, McGuinness G, McCauley DI, Miettinen OS (2002) CT screening for lung cancer: frequency and significance of part-solid and nonsolid nodules. AJR 178:1053-1057

26. Naidich DP, Bankier AA, MacMahon H, Schaefer-Prokop CM, Pistolesi M, Goo JM, Macchiarini P, Crapo JD, Herold CJ, Austin JH, Travis WD (2013) Recommendations for the managemen of subsolid pulmonary nodule detected at CT: a statement from the Fleischner Society. Radiol 266(1):304-317

27. Godoy MC, Naidich DP (2012) Overview and strategic management of subsolid pulmonary nodules. J Thorac Imaging 27:240-248

28. Wahidi MM, Govert JA, Goudar RK, Gould MK, McCrory DC (2007) American College of Chest Physicians. Evidence for the treatment of patients with pulmonary nodules: when is it lung cancer ?: ACCP evidence-based clinical practice guidelines (2nd edition). Chest 132(3 Suppl):94S-107S

29. Bellomi M, Veronesi G, Rampinelli C, Ferretti S, De Fiori E, Maisonneuve P (2007) Evolution of lung nodules 5or $1 / 45 \mathrm{~mm}$ detected with low-dose CT in asymptomatic smokers. Br J Radiol 80: 708-712

30. Veronesi G, Bellomi M, Scanagatta P et al (2008) Difficulties encountered managing nodules detected during a computed tomography lung cancer screening program. J Thorac Cardiovasc Surg 136: 611-617

31. Horeweg N, van der Aalst CM, Thunnissen E, Nackaerts K, Weenink C (2013) Characteristics of lung cancers detected by computer tomography screening in the randomized NELSON trial. Am J Respir Crit Care Med 187(8):848-854

32. Prichard MG, Brown PG, Sterrett GF (1984) Bronchioloalveolar carcinoma arising in longstanding lung cysts. Thorax 39:545-549

33. Zulueta JJ, Bloom SM, Rozansky MI et al (1996) Lung cancer in patients with bullous disease. Am J Respir Crit Care Med 154:519 522

34. Maki D, Takahashi M, Murata K et al (2006) Computed tomography appearances of bronchogenic carcinoma associated with bullous lung disease. J Comput Assist Tomogr 30:447-452

35. Mascalchi M, Attinà D, Bertelli E, Falchini M, Vella A, Pegna AL, Ambrosini V, Zompatori M (2015) Lung cancer associated with cystic airspaces. J Comput Assist Tomogr 39(1):102-108

36. Farooqi AO, Cham M, Zhang L, Beasley MB, Austin JH, Miller A, Zulueta JJ, Roberts H, Enser C, Kao SJ, Thorsen MK, Smith JP, Libby DM, Yip R, Yankelevitz DF, Henschke CI (2012) International Early Lung Cancer Action Program Investigators. Lung cancer associated with cystic airspaces. AJR Am J Roentgenol 199(4):781-786

37. Scholten ET, Jacobs C, van Ginneken B, van Riel S, Vliegenthart R, Oudkerk M (2015) Detection and quantification of the solid component in pulmonary subsolid nodules by semiautomatic segmentation. Eur Radiol 25(2):488-496

38. Devaraj A (2015) Missed cancers in lung cancer screening-more than meets the eye. Eur Radiol 25(1):89-91

39. Mascalchi M, Picozzi G, Falchini M, Vella A, Diciotti S, Carrozzi L, Pegna AL, Falaschi F (2014) Initial LDCT appearance of incident lung cancers in the ITALUNG trial. Eur J Radiol 83(11):2080-2086

40. Xu DM, Yip R, Smith JP, Yankelevitz DF, Henschke CI (2014) IELCAP Investigators. Retrospective review of lung cancers diagnosed in annual rounds of CT screening. AJR Am J Roentgenol 203(5):965-972

41. Gietema HA, Wang Y, Xu D et al (2006) Pulmonary nodules detected at lung cancer screening: interobserver variability of semiautomated volume measurements. Radiol 241:251-256. doi: 10.1148/radiol.2411050860

42. Truong MT, Ko JP, Rossi SE, Rossi I, Viswanathan C, Bruzzi JF, Marom EM, Erasmus JJ et al (2014) Update in the Evaluation of the Solitary Pulmonary Nodule. RadioGraphics 34:1658-1679

43. Heuvelmans MA, Oudkerk M, de Bock GH et al (2013) Optimisation of volume-doubling time cutoff for fast-growing lung 
nodules in CT lung cancer screening reduces false-positive referrals. Eur Radiol 23:1836-1845

44. MacMahon H, Austin JH, Gamsu G et al (2005) Guidelines for management of small pulmonary nodules detected on CT scans: a statement from the Fleischner Society. Radiol 237:395-400

45. Lindell RM, Hartman TE, Swensen SJ, Jett JR, Midthun DE, Tazelaar HD, Mandrekar JN (2007) Five-year lung cancer screening experience: $\mathrm{CT}$ appearance, growth rate, location, and histologic features of 61 lung cancers. Radiol 242(2):555-562

46. Scholten ET, Horeweg N, De Koning HJ, Vliegenthart R, Oudkerk M, Mali WPTM et al (2015) Computed tomographic characteristics of interval and post screen carcinomas in lung cancer screening. Eur Radiol 25(1):81-88

47. Li F, Sone S, Abe H, MacMahon H, Armato SG, Doi K (2002) Lung cancers missed at low-dose helical CT screening in a general population: comparison of clinical, histopathologic, and imaging indings. Radiol 225(3):673-683

48. Henschke CI, Yankelevitz DF, Yip R, Reeves AP, Farooqi A, Xu D, Smith JP, Libby DM, Pasmantier MW, Miettinen OS, Writing Committee for the I-ELCAP Investigators (2012) Lung cancers diagnosed at annual CT screening: volume doubling times. Radiol 263(2):578-583
49. Diederich S, Wormanns D, Semik M, Thomas M, Lenzen H, Roos N, Heindel W (2002) Screening for early lung cancer with low-dose spiral CT: prevalence in 817 asymptomatic smokers. Radiol 222(3): 773-781

50. Sverzellati N, Guerci L, Randi G, Calabrò E, La Vecchia C, Marchianò A, Pesci A, Zompatori M, Pastorino U (2011) Interstitial lung diseases in a lung cancer screening trial. Eur Respir J 38(2):392-400

51. Van de Wiel JC, Wang Y, Xu DM et al (2007) Neglectable benefit of searching for incidental findings in the Dutch-Belgian lung cancer screening trial (NELSON) using low-dose multidetector CT. Eur Radiol 17:1474-1482

52. Oh SY, Kim MY, Kim JE, Kim SS, Park TS (2015) Evolving Early Lung Cancers Detected During Follow-Up of Idiopathic Interstitial Pneumonia: Serial CT Features. AJR Am J Roentgenol 204(6): 1190-1196

53. Fraire AE, Greenberg SD (1973) Carcinoma and diffuse interstitial fibrosis of lung. Cancer 31:1078-1086

54. Kishi K, Homma S, Kurosaki A, Motoi N, Yoshimura K (2006) High-resolution computed tomography findings of lung cancer associated with idiopathic pulmonary fibrosis. J Comput Assist Tomogr 30:95-99 